\title{
Supplemental Files: Modelling Responses of the Inert-gas Washout and MRI to Bronchoconstriction
}

\author{
Brody H. Foy*, David Kay, Rafel Bordas \\ Department of Computer Science, University of Oxford, Oxford, Oxfordshire, United \\ Kingdom
}

\section{Supplemental 1: Details of the numerical solution scheme}

Solution scheme for the flow-pressure model

The model for flow, pressure and acinar region volume expansion and contraction comprises the equations

$$
\begin{aligned}
\Delta P & =R(Q) Q, & \text { for each branch, } \\
Q_{\text {upper }} & =\sum Q_{\text {lower }}, & \text { for each bifurcation, } \\
\frac{d}{d t} V_{\text {acin }, i} & =Q_{i}, & \text { for each terminal branch, } \\
P_{T, i}-P_{p l} & =\frac{1}{C_{i}} V_{\text {acin }, i}, & \text { for each terminal branch, }
\end{aligned}
$$

where $R(Q)$ is the Poiseuille resistance function, with a Pedley correction term:

$$
R(Q)=\frac{32 \mu L c}{\pi d^{4}}\left(\operatorname{Re}(Q) \frac{d}{L}\right)^{1 / 2}
$$

where $L$ and $d$ are the branch length and diameter, $\mu$ is the air viscosity, $\operatorname{Re}(Q)$

5 is the Reynold's number of the flow, and $c=1.85$ is a constant.

The pleural pressure was taken as a temporal sinusoidal function with magnitude chosen to enforce tidal breathing of 1 Litre per breath cycle, and the compliance was dispersed according to the gravitational gradient outlined in

\footnotetext{
* Corresponding author

Email address: brody.foy@new.ox.ac.uk (Brody H. Foy*)
} 
the Materials and Methods. To close the system, an atmospheric pressure condition $P=0$ was applied at the trachea.

To solve this system numerically, a Backward Euler discretisation was used for the temporal derivative. The timestep was chosen as $d t=0.01$, which was small enough that further stepsize reductions caused solution changes of less than $1 \%$. This generated a large $(\approx 200,000$ variables $)$, stiff, non-linear equation system. The system was solved using a standard vector Newton Method, with the Jacobian being constructed through difference approximations. The Jacobian was built infrequently, only being updated if a Newton iteration took more than 10 steps to converge. This allowed for efficient solution of the matrix system using LU factorisation.

\section{Solution of the gas transport model}

Once flow rates had been calculated, we discretised the transport model

$$
\frac{\partial A C}{\partial t}=-u \frac{\partial A C}{\partial x},
$$

using a finite volume scheme, with a single node centred at each bifurcation point, and one node at the centre of each acinar region (an example schematic is given in figure 1). Along each branch a variable number of nodes were placed, 25 with the trachea having the most (8), and the terminal conducting zone branches having the least (1). The total number of control volumes was increased until further increases caused solution changes of less than $1 \%$. This led to a system size of approximately 300000 variables.

Applying the finite volume scheme, for a control volume $V_{i}$, with gas concentration $C_{i}$, and average cross-sectional $A_{i}$ we have:

$$
\left|V_{i}\right| \frac{\partial A_{i} C_{i}}{d t}=\sum_{j \in S_{i}}\left(J_{i, j} \cdot \hat{n}_{i, j}\right) A_{i, j},
$$

where $S_{i}$ is the set of all control volumes that share a boundary with $V_{i}, \hat{n}_{i, j} \in$ $(1,-1)$ is a unit normal pointing outward from $V_{i}$ towards $V_{j}, A_{i, j}$ is the cross- 


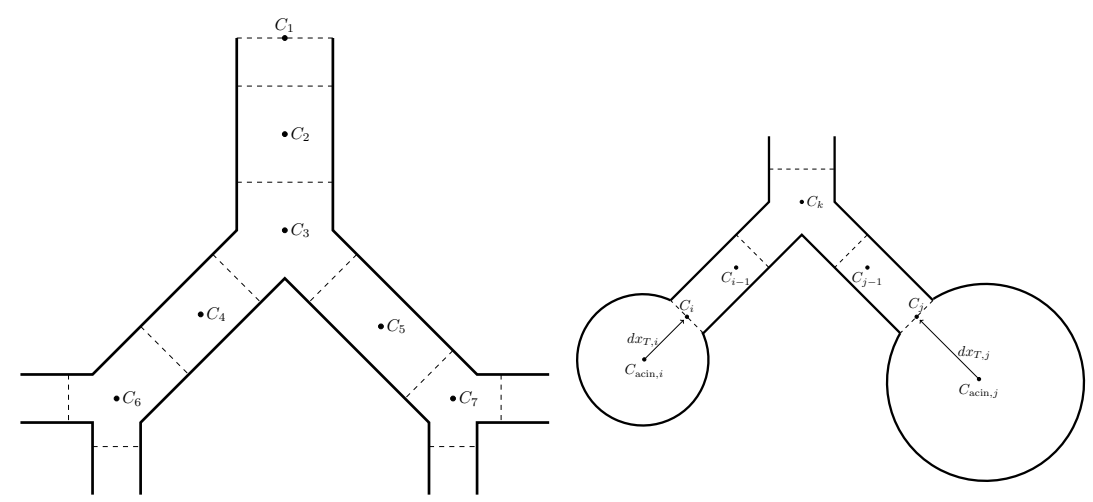

Figure 1: Schematic of finite volume discretisation. An idealised 2D cross-section of the problem domain, showing the finite volume discretisation of branches (left) and of the acinar regions (right). Control volume boundaries (dashed lines) are spaced evenly between nodes, except at the domain boundaries.

sectional area of the boundary, and the boundary flux $J_{i, j}$ is

$$
J_{i, j}=-\left.(u A C)\right|_{\partial V_{i, j}},
$$

with $\partial V_{i, j}$ denoting the boundary of $V_{i}$ and $V_{j}$. To approximate the convection 35 terms we use an upwinding and downwinding scheme such that

$$
J_{i, j} \approx-u A_{i, j} C_{i}
$$

when flow is from node $i$ to node $j$, and

$$
J_{i, j} \approx-u A_{i, j} C_{j}
$$

when flow is from node $j$ to node $i$.

At the upper boundary, we applied a no flux boundary condition during inhalation, and the condition

$$
J_{1,0}=u A_{1,0} C_{1}
$$

40 during exhalation.

At the connection of the terminal conducting zone branches to the acinar regions, mass transfer is driven by the same convection discretisation as shown 
in equation 2. Due to the changing volumes of the acinar regions, gas concentration transfer could not be directly calculated. Instead, mass changes were calculated, and then concentrations recalculated based on the new volumes for that timestep.

To close the system, we specified the initial condition

$$
C(x, 0)=C_{0},
$$

which assumes that the simulation begins at the start of the washout phase, meaning the gas concentration has equilibrated within the lungs.

To discretise the temporal derivative in equation2, a Backward Euler method was applied, with $d t=0.01$. The resulting matrix system was solved in MATLAB using LU factorisation, with approximate minimum degree reordering, and sparse storage.

A list of key parameters used in the numerical scheme (and relating to the physical model) are given in table 1 .

\begin{tabular}{l|lr} 
Parameter & Symbol (units) & Value \\
\hline Air density & $\rho\left(\mathrm{kg} \cdot \mathrm{m}^{-3}\right)$ & 1.15 \\
Air viscosity & $\mu\left(\mathrm{kg} \cdot \mathrm{m}^{-1} \cdot \mathrm{s}^{-1}\right)$ & $1.92 \times 10^{-5}$ \\
Time stepsize & $d t(\mathrm{~s})$ & 0.01 \\
Initial gas concentration & $C_{0}(\%)$ & 4
\end{tabular}

Table 1: A table of parameters used in the numerical scheme

\section{Supplemental 2: Presentation of key results against branch genera- tion}

Within the manuscript we chose to use Strahler order (the number of branches between a point and the nearest terminal bronchiole) as the marker of depth, instead of branch generation (the number of branches between a point and the trachea). For completeness, in this supplemental we present some of the key results from the manuscript, using branch generation as the depth marker. 
The simulation protocol was similar to the process outlined in section 2.4. However, for each simulation, a branch generation (3-16) was uniformly random drawn instead of a Strahler order. The randomly drawn constriction severity (0$99 \%$ ) was applied in a spatially coherent manner (as outlined in section 2.4) to a randomly selected $20 \%$ of branches of that generation. For reference, the trachea has branch generation 0 , and terminal bronchioles have branch generations from $13-16$.

In Fig 2 we give plots of $s_{\text {cond }}$, LCI, $\sigma_{r}$ and $\sigma_{\text {local }}$ against constriction severity. Within this figure we see the same trends as exhibited in Fig 1 in the manuscript. The only significant difference is that $\sigma_{\text {local }}$ has a smaller peak value when constricting by branch generation than when constricting by Strahler order. This is most likely due to less branches being constricted overall, as since there 75 are 16 branch generations but only 12 Strahler orders, $20 \%$ of branches of a given generation represents a smaller overall share of the airway tree.

Within Fig 3 we give plots of $s_{\text {cond }}$, LCI, $\sigma_{r}$ and $\sigma_{\text {local }}$ against branch generation. Results have been separated into six bands of constriction severity: $<50 \%, 50-75 \%, 75-85 \%, 85-90 \%$ 90-95\%, 95-100\%. Comparative to Fig 3 in the manuscript, the overarching trends are the same, with the direction reversed, due to the fact that high Strahler order and low generation both represent proximal depth. The response when marking depth with Strahler order appears to be slightly less noisy, however, this effect is quite mild. As can be seen, Fig 3 still clearly illustrates that $\sigma_{\text {local }}$ has the strongest and most consistent response 85 to constriction depth of the four indices. 

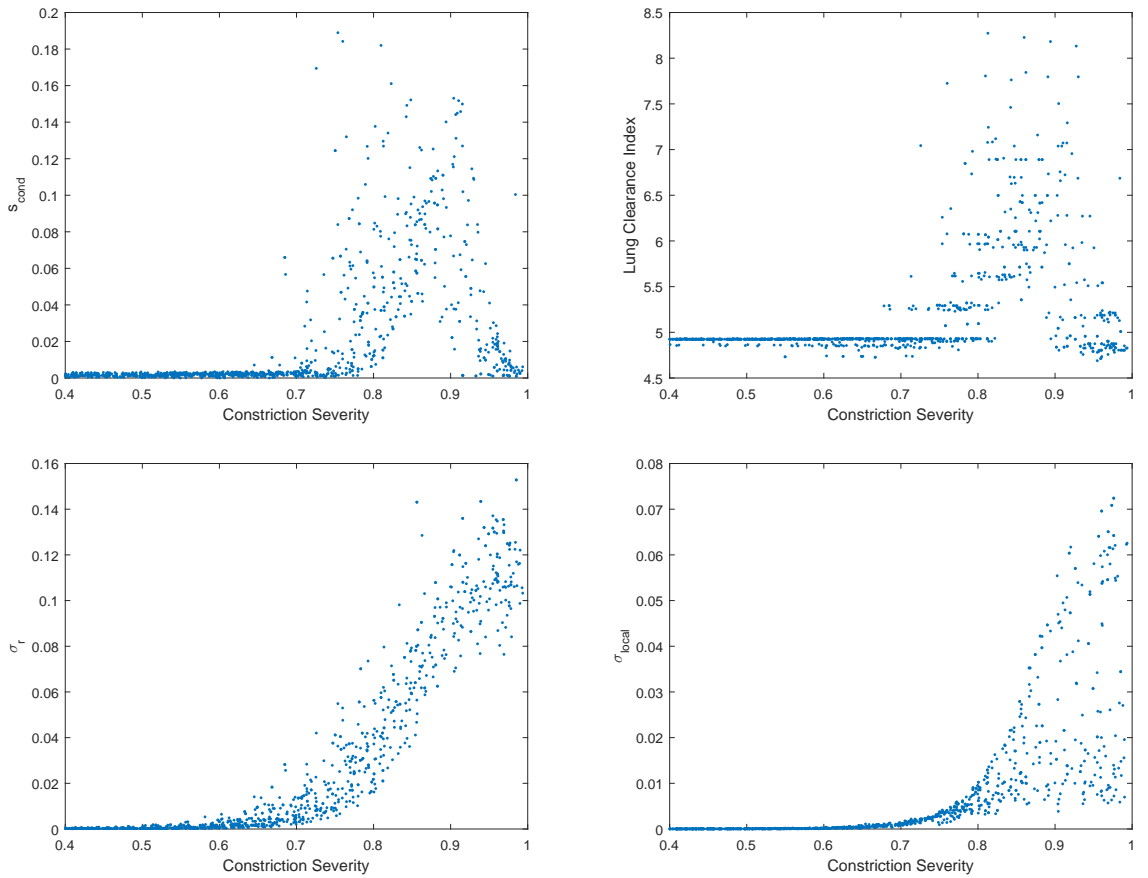

Figure 2: Variation of $s_{\text {cond }}$, LCI, $\sigma_{r}$ and $\sigma_{\text {local }}$ against constriction severity. Positive responses are initially seen from all four indices, followed by a decrease to baseline in the MBW indices as severity approaches airway closure. The variance of $\sigma_{\text {local }}$ is also seen to be significantly larger than that of the other indices. 

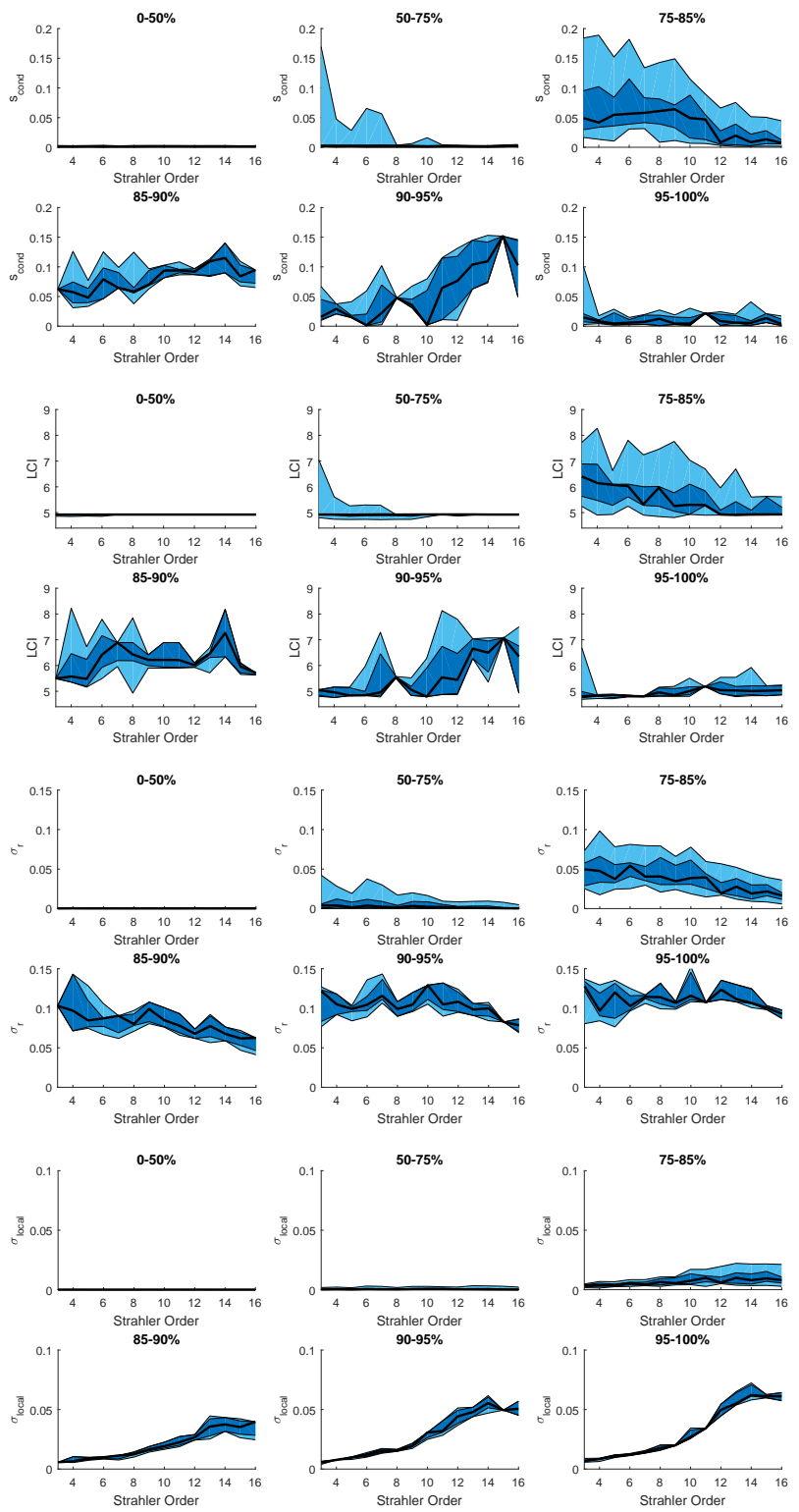

Figure 3: Variation of $s_{\text {cond }}$, LCI, $\sigma_{r}$ and $\sigma_{\text {local }}$ against constriction depth (Branch generation). Results have been separated into six different constriction severity bands. For each band, the median output (line) is presented alongside an inner band representing the interquartile range (dark blue), and an outer band to the minimum and maximum values (light blue). The MBW indices show no consistent correlation with depth, but the MR indices respond more clearly, with $\sigma_{\text {local }}$ having the strongest and most consistent response. 